Received: 20 November 2014, Accepted: 29 June 2015

Edited by: C. A. Condat, G. J. Sibona

Reviewed by: A. De Luca, Laboratoire de Physique Theorique,

ENS \& Institut Philippe Meyer, Paris, France.

Licence: Creative Commons Attribution 3.0

DOI: http://dx.doi.org/10.4279/PIP.070012 www.papersinphysics.org

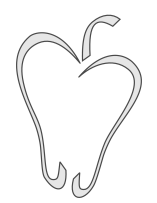

ISSN 1852-4249

\title{
Role of energy uncertainties in ergodicity breaking induced by competing interactions and disorder. A dynamical assessment through the Loschmidt echo.
}

\author{
Pablo R. Zangara, ${ }^{1,2 *}$ Patricia R. Levstein, ${ }^{1,2}$ Horacio M. Pastawski ${ }^{1,2 \dagger}$
}

\begin{abstract}
A local excitation in a quantum many-particle system evolves deterministically. A timereversal procedure, involving the invertion of the signs of every energy and interaction, should produce an excitation revival: the Loschmidt echo (LE). If somewhat imperfect, only a fraction of the excitation will refocus. We use such a procedure to show how noninverted weak disorder and interactions, when assisted by the natural reversible dynamics, fully degrade the LE. These perturbations enhance diffusion and evenly distribute the excitation throughout the system. Such a dynamical paradigm, called ergodicity, breaks down when either the disorder or the interactions are too strong. These extreme regimes give rise to the well known Anderson localization and Mott insulating phases, where quantum diffusion becomes restricted. Accordingly, regardless of the kinetic energy terms, the excitation remains mainly localized and out-of-equilibrium, and the system behaves non-ergodically. The LE constitutes a fair dynamical witness for the whole phase diagram since it evidences a surprising topography in which ergodic and non-ergodic phases interpenetrate each other. Furthermore, we provide an estimation for the critical lines separating the ergodic and non-ergodic phases around the Mott and Anderson transitions. The energy uncertainties introduced by disorder and interaction shift these thresholds towards stronger perturbations. Remarkably, the estimations of the critical lines are in good agreement with the phase diagram derived from the LE dynamics.
\end{abstract}

\section{Introduction}

According to Classical Mechanics, a system composed by $N$ particles in $d$ dimensions is described as a point $X$ in a $(2 d N)$-dimensional phase space. If the system is conservative, the energy is the primary conserved quantity, and the phase space is

\footnotetext{
*Email: zangara@famaf.unc.edu.ar

$\dagger^{\dagger}$ Email: horacio@famaf.unc.edu.ar

1 Instituto de Física Enrique Gaviola (CONICET-UNC), Argentina.

2 Facultad de Matemática, Astronomía y Física, Universidad Nacional de Córdoba, 5000 Córdoba, Argentina.
}

restricted to a hypersurface $\mathcal{S}$ of $2 d N-1$ dimensions usually called energy shell. Fully integrable systems are further constrained, and their solutions turn out to be regular and non-dense periodic orbits contained in $\mathcal{S}$. If integrability is broken, the orbits become irregular and cover $\mathcal{S}$ densely. This means that the actual trajectory $X(t)$ will uniformly visit every configuration within $\mathcal{S}$, provided that enough time has elapsed. This last observation embodies the concept of ergodicity: an observable can be equivalently evaluated by averaging it for different configurations in $\mathcal{S}$ or by its time-average along a single trajectory $X(t)$. In such a sense, ergodicity sets the equivalence between the Gibbs' description 
Papers in Physics, vol. 7, ART. 070012 (2015) / P. R. Zangara et al.

in terms of ensembles and Boltzmann kinetic approach to Thermostatistics. Therefore, the ergodic hypothesis has become the cornerstone of Classical Statistical Mechanics [1,2].

Almost 60 years ago, E. Fermi, J. Pasta and S. Ulam (FPU) [3] tried to study when and how the integrability breakdown can lead to an ergodic behavior within a deterministic evolution. They considered a string of harmonic oscillators perturbed by anharmonic forces in order to verify that these non-linearities can lead to energy equipartition as a manifestation of ergodicity. Even though Ulam himself stated "The motivation then was to observe the rates of mixing and thermalization..." [4], the results were not the expected ones: "thermalization" dynamics did not show up at all. Nowadays, their striking results are well understood in terms of the theory of chaos [5]. In this context, chaos is defined as an exponential sensitivity to changes in the initial condition. In fact, the onset of dynamical chaos $[6,7]$ can be identified with the transition from non-ergodic to ergodic behavior. Therefore, within classical physics, the emergence of ergodicity can be satisfactorily explained [8].

The previous physical picture cannot be directly extended to Quantum Mechanics. Indeed, any closed quantum system involves a discrete energy spectrum and evolves quasi-periodically in the Hilbert space, which becomes the quantum analogue to the classical phase space. Nevertheless, thermalization and ergodicity in isolated quantum systems could still be defined for a set of relevant observables $[9,10]$. Since the sensitivity to initial conditions does not apply to quantum systems, the quantum signature of dynamical chaos had to be found as an instability of an evolution towards perturbations in the Hamiltonian [11]. Because this definition encompasses the classical one, it builds a bridge between classical and quantum chaos. Moreover, since it also implies an instability towards perturbations in a time reversal procedure, it can be experimentally evaluated [12] as the amount of excitation recovered or Loschmidt echo (LE) $[13,15]$. Such a revival is degraded by the presence of uncontrolled environmental degrees of freedom as in the usual picture of decoherence for open quantum systems [14]. Strikingly, in closed systems with enough internal complexity, even simple perturbations seem to degrade the LE in a time scale given by the reverted dynamics, revealing how a mixing dynamics drives irreversibility $[16,17]$.

Within the last years, a new generation of experiments on relaxation and equilibration dynamics of (almost perfectly) closed quantum many-particle systems has become accessible employing optical lattices loaded with cold atoms $[18,19]$. These became the driving force behind the recent theoretical efforts to grasp quantum thermalization [20].

Attempting a step beyond the FPU problem, the current aim is to study simple quantum models that could go parametrically from an ergodic to a non-ergodic quantum dynamics. Moreover, a fundamental question is whether such a transition occurs as a smooth crossover or a sharp threshold. A promising candidate for these studies would be a system showing Many-Body Localization (MBL) $[21,22]$. This dynamical phenomenon occurs when an excitation in a disordered quantum system evolves in presence of interactions. In fact, the MBL is a quantum dynamical phase transition between extended and localized many-body states that results from the competition between interactions [23] and Anderson disorder [24]. If the many-body states are extended, then one may expect that the system is ergodic enough to behave as its own heat bath. In such case, single energy eigenstates would yield expectation values for few-body observables that coincide with those evaluated in the microcanonical thermal ensemble $[25,26]$. Quite on the contrary, if the many-body states are localized, any initial out-of-equilibrium condition would remain almost frozen. In this case, self-thermalization is precluded. Therefore, the MBL would evidence the sought threshold between ergodic and non-ergodic behavior.

In this article, we address the competition between interactions and disorder in a onedimensional (1D) spin system. It is already known that such models evidence the MBL transition, at least for particular parametric regimes [27-31]. Our approach to tackle this problem involves the evaluation of the LE, here defined as the amount of a local excitation recovered after an imperfect time reversal procedure. This involves the inversion of the sign of the kinetic energy terms in the many-spin Hamiltonian [14]. Moreover, the LE is evaluated as an autocorrelation function that could become a suitable order parameter [32]. Thus, the LE is a natural observable that allows us to identify when the ergodicity of an excitation dynamics is broken 
Papers in Physics, vol. 7, ART. 070012 (2015) / P. R. Zangara et al.

as interactions and disorder become strong enough. When weak, these "perturbations" favor the excitation spreading, but limit LE recovery as they are not reversed.

From the actual LE time-dependence in the infinite-temperature regime, we extract a dynamical phase diagram that shows a non-trivial interplay between interactions and disorder. The near-zero temperature regime has already been addressed in the literature $[33,34]$ and there are conjectures about the global topography of the phase diagram [35]. In analogy with this last case, we address the nature of two critical lines that separate the ergodic phase from two different non-ergodic phases: the Mott insulator and the MBL phase. The appearance of either, Mott insulator and MBL phases, can be well estimated in terms of the relevant energy scales. Thus, in order to evaluate an estimation of the critical lines, we compute the energy uncertainties that weak disorder and weak interactions would impose on the states involved in the Mott transition and on the MBL transition, respectively. Quite remarkably, these estimations show a good agreement with the dynamical LE diagram. Our approach allows the identification of ergodic and non-ergodic phases whose non-trivial structure may guide future theoretical and experimental investigations.

\section{Loschmidt echo formulation}

We consider a $1 \mathrm{D}$ spin system that evolves according to a Hamiltonian $\hat{H}=\hat{H}_{0}+\hat{\Sigma}$. Here, $\hat{H}_{0}$ stands for a nearest neighbors $X Y^{1}$ Hamiltonian [36, 37]:

$$
\begin{aligned}
\hat{H}_{0} & =\sum_{i=1}^{N} J\left[S_{i}^{x} S_{i+1}^{x}+S_{i}^{y} S_{i+1}^{y}\right] \\
& =\sum_{i=1}^{N} \frac{1}{2} J\left[S_{i}^{-} S_{i+1}^{+}+S_{i}^{+} S_{i+1}^{-}\right]
\end{aligned}
$$

which because of the periodic boundary conditions can be thought as arranged in a ring. Here, unless explicitly stated, $N=12$. Notice that $\hat{H}_{0}$ can be mapped into two independent non-interacting

\footnotetext{
${ }^{1}$ Notice that, in the recent literature of stronglycorrelated systems within the condensed matter community, the notation $X X$ is employed instead of $X Y$.
}

fermion systems by the Wigner-Jordan transformation [38]. Therefore, it encloses fully integrable single-particle dynamics.

The integrability of the model is broken by the Ising interactions and the on-site disorder enclosed in $\hat{\Sigma}$,

$$
\hat{\Sigma}=\sum_{i=1}^{N} \Delta S_{i}^{z} S_{i+1}^{z}+\sum_{i=1}^{N} h_{i} S_{i}^{z},
$$

where $\Delta$ is the magnitude of the homogeneous interaction and $h_{i}$ are randomly distributed fields in the range $[-W, W]$. In order to enable the comparison with the standard Anderson localization literature, we stress that $W$ here is half of the standard strength commonly used for the Anderson disorder [39].

The initial out-of-equilibrium condition is given by an infinite-temperature state in which a local excitation (polarization) is injected at site 1 :

$$
\left|\Psi_{n e q}\right\rangle=\left|\uparrow_{1}\right\rangle \otimes\left\{\sum_{r=1}^{2^{N-1}} \frac{1}{\sqrt{2^{N-1}}} e^{\mathrm{i} \varphi_{r}}\left|\beta_{r}\right\rangle\right\}
$$

where $\varphi_{r}$ is a random phase and $\left\{\left|\beta_{r}\right\rangle\right\}$ are state vectors in the computational Ising basis of the $N-1$ remaining spins. The state defined in Eq. (4) is a random superposition over the whole Hilbert space, and can successfully mimic the dynamics of ensemble calculations [40]. Additionally, notice that $\hat{\Sigma}$ perturbs the quantum phase of each of the Ising states participating in the superposition.

Two evolution operators are built from the Hamiltonian operators 1 and 3 , according to the relative sign between $\hat{H}_{0}$ and $\hat{\Sigma}$. These are $\hat{U}_{+}\left(t_{R}\right)=$ $\exp \left[-\frac{\mathrm{i}}{\hbar}\left(\hat{H}_{0}+\hat{\Sigma}\right) t_{R}\right]$ and $\hat{U}_{-}\left(t_{R}\right)=\exp \left[-\frac{\mathrm{i}}{\hbar}\left(-\hat{H}_{0}+\right.\right.$ $\left.\hat{\Sigma}) t_{R}\right]$. In this scenario, the LE is defined as the revival of the local polarization at site 1 :

$$
\begin{aligned}
& M_{1,1}(2 t) \\
& =2\left\langle\Psi_{n e q}\left|\hat{U}_{+}^{\dagger}(t) \hat{U}_{-}^{\dagger}(t) \hat{S}_{1}^{z} \hat{U}_{-}(t) \hat{U}_{+}(t)\right| \Psi_{n e q}\right\rangle .
\end{aligned}
$$

It is important to stress that Eq. (5) constitutes, at least in principle, an actual experimental observable of the kind evaluated since the early LE experiments $[12,16,17]$, see also Ref. [41]. Moreover, both the local excitation and detection are well established techniques within solid-state NMR 
[42]. Nevertheless, changing the signs of specific Hamiltonian terms results in a more subtle task. While the standard dipole-dipole interaction can be reverted [43], the planar $X Y$ interaction requires much more sophisticated pulse sequences, even for its forward implementation [37]. In particular, the mapping of the $X Y$ interaction into a double-quantum Hamiltonian, strictly valid in 1D systems [44], could provide a novel approach to the problem of localization as it recently did for $3 \mathrm{D}$ systems [45]. This could become a pathway towards an experimental realization much related to the problem considered here.

In order to analyze the ergodicity of our observable, we evaluate the mean LE, $\bar{M}_{1,1}$ :

$$
\bar{M}_{1,1}(T)=\frac{1}{T} \int_{0}^{T} M_{1,1}(t) d t .
$$

The standard analysis of localization implies the computation of $\lim _{T \rightarrow \infty} \bar{M}_{1,1}(T)$. However, since the LE is evaluated within a finite system, dynamical recurrences known as Mesoscopic echoes show up at the (single-particle) Heisenberg time $T_{H}$ of $\hat{H}_{0}$. As extensively discussed in Refs. $[36,38,46]$, $T_{H}$ can be estimated as

$$
T_{H} \sim 2 \sqrt{2} N \frac{\hbar}{J}
$$

This estimation can be interpreted as the time needed by a local excitation to wind around a ring of length $L=N \times a$ at an average speed $v_{M} / \sqrt{2}$, with a maximum group velocity $v_{M}=a \times \frac{1}{2} \mathrm{~J} / \hbar$. Since these recurrences are spurious to our analysis of the limiting case $N \rightarrow \infty$, we restrict our analysis to $T<T_{H}$.

Let us provide some specific details that should allow the reproduction of our numerical computation. We evaluate Eq. (6) ranging both $\Delta$ and $W$ within the interval $[0,5 J]$, considering increments of $0.2 J$ in both magnitudes. The relevant parameter regions were explored in more detail by employing steps of $0.1 \mathrm{~J}$. For each parameter set, 10 realizations of disorder were averaged, each of them with second moment $\left\langle h^{2}\right\rangle=W^{2} / 3$. For the noninteracting case $\Delta=0$, i.e., pure Anderson disorder, we computed 500 disorder realizations. With the purpose of keeping the statistical fluctuations negligibly small, an extra average over 10 realizations of $\left|\Psi_{n e q}\right\rangle$ is performed by tossing the phases $\varphi_{r}$ in the whole $[0,2 \pi)$ range.

\section{A dynamical phase diagram}

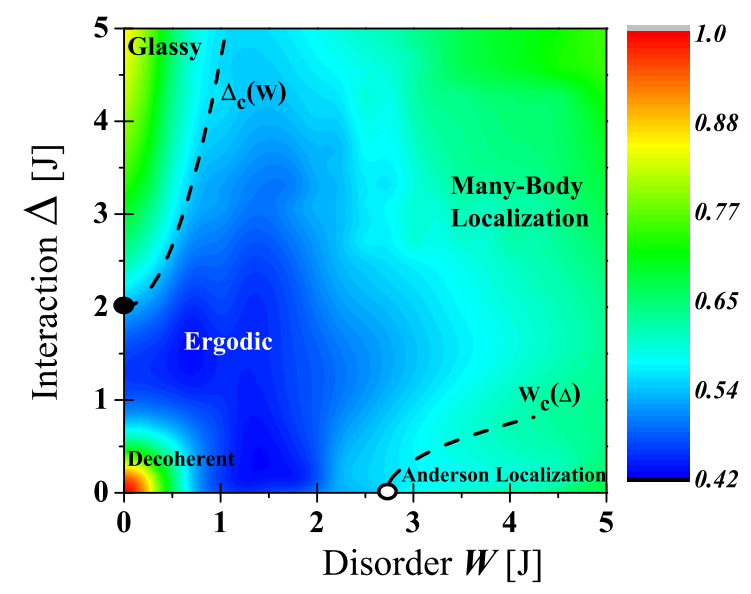

Figure 1: Dynamical phase diagram: $\bar{M}_{1,1}(T)$ level plot at $T=12 \hbar / J$ as a function of the interaction strength $\Delta$ and disorder $W$.

Figure 1 displays the dynamical phase diagram for the LE. It is given by a level plot of $\bar{M}_{1,1}$ at $T=$ $12 \hbar / J$, as function of the interaction $\Delta$ and disorder strength $W$. Within the diagram, five dynamical regions are identified according to the predominant mechanism: decoherent, ergodic, glassy, Anderson localization, and Many-Body Localization.

If both $\Delta$ and $W$ are weak, the system is almost reversible, the dynamics is controlled by single particle propagations and therefore $\bar{M}_{1,1}$ remains near 1. This means that despite of the slight phase perturbations, the local excitation can be driven back by the reversal of $\hat{H}_{0}$. Thus, the parametric region at the bottom left corner may be associated with decoherence, i.e., a sort of spin wave behavior weakly perturbed by the imperfect control of the internal degrees of freedom $[37,47]$.

If either $\Delta$ or $W$ are further increased, the propagation of a local excitation, ruled by $\hat{H}_{0}$, suffers the effects of $\hat{\Sigma}$ as multiple scattering events with the disordered potential and with other spins. Thus, the excitation enters in a diffusive regime where it rapidly spreads all over the spin system. As these scattering processes cannot be undone by the reversal procedure, the spreading becomes irreversible. Consistently, this bluish region is associated with an ergodic behavior for the polarization. In this 
regime, the polarization becomes evenly distributed within the system, i.e., $2\left\langle\hat{S}_{j}^{z}\right\rangle=1 / N$ for all $j$. The ideal limit $\bar{M}_{1,1}(T \rightarrow \infty) \rightarrow 1 / N$ is verified up to a numerical offset that comes from the transient decay of the LE.

If $W=0$, a strong increase in $\Delta$ leads to a predominance of the Ising interaction, which freezes the polarization dynamics. Since the quantum diffusion induced by $\hat{H}_{0}$ results drastically constrained, $\bar{M}_{1,1}$ remains trivially high. We interpret such behavior as a glassy dynamics with long relaxation times. In fact, this sort of localization corresponds to the Mott insulating phase of an impurity band [23]. Additionally, the color contrast around $\Delta \gtrsim 2 J$ suggests that the glassy-ergodic transition remains abrupt even for nonzero disor$\operatorname{der}(W \lesssim 1.0 J)$. This indicates a parameter region where the interaction-disorder competition leads to a sharp transition between the glassy and the ergodic phases. However, since transient phenomena become very slow, a reliable finite size scaling of this regime would require excessively long times to capture how a vitreous dynamics is affected by disorder.

A dimensional argument provides a hint on the nature of the critical line that separates the ergodic and glassy phases. In fact, the Mott transition typically occurs when the interaction strength $\Delta$ is comparable to the bandwidth $B=2 J$. Such a particular interaction strength is singled out in Fig. 1 by a full black circle. Adding a weak disorder introduces an energy uncertainty $\delta E$ on the energy levels that would widen $B$. In order to estimate it, we resort to its corresponding time scale $\tau$, which in turn can be evaluated according to the Fermi golden rule (FGR). With such a purpose, we consider a localized excitation that can "escape" either to its right or to its left side, where two semi-infinite linear chains are symmetrically coupled. Then,

$$
\frac{1}{\tau}=2 \frac{2 \pi}{\hbar}\left(\frac{W^{2}}{3}\right) N_{1}(\varepsilon) .
$$

Here, as stated above, $W^{2} / 3$ stands for second moment of the disorder distribution. The factor 2 accounts for the two alternative decays (right and left). Additionally, $N_{1}(\varepsilon)$ is the Local Density of States (LDoS) of a semi-infinite linear chain with hopping element $J / 2$,

$$
N_{1}(\varepsilon)=\frac{2}{\pi J} \sqrt{1-\left(\frac{\varepsilon}{J}\right)^{2}} .
$$

The energy levels acquire a Lorentzian broadening which, evaluated at the spectral center $\varepsilon=0$, results

$$
\delta E=\frac{\hbar}{2 \tau}=\left.\frac{4}{3} \frac{W^{2}}{J} \sqrt{1-\left(\frac{\varepsilon}{J}\right)^{2}}\right|_{\varepsilon=0}=\frac{4}{3} \frac{W^{2}}{J} .
$$

Since half of the states lie beyond the range $B+$ $2 \delta E$, one may attempt an estimation of the critical line for the Mott transition as,

$$
\Delta_{c}(W) \sim B+2 \delta E \sim 2 J+\frac{8}{3} \frac{W^{2}}{J},
$$

which is displayed in Fig. 1 as a dashed line.

A similar functional dependence as the one discussed here for the glassy-ergodic interphase was conjectured by Kimball for the interacting ground state diagram [35]. Additionally, it is worthy to mention that a naive expectation about the morphology of the phase diagram with two competing magnitudes would be a semi-circular shape. This is precisely the case of magnetic field and temperature as in the phase diagram of a type I superconductor. Thus, one of the highly non-trivial implications of the reentrance of the ergodic phase at large $\Delta$ in our diagram is to debunk such an expectation.

If $\Delta=0$, the picture for $W>0$ is the standard Anderson Localization problem. Here, a reliable estimate of the localization length is only possible when it is smaller than the finite size of the system. Not being this the case of very weak disorder, the LE degrades smoothly as a function of time with a dynamics that cannot be distinguished from a diffusive one. When the disorder is strong enough, the localization length becomes comparable with the lattice size and thus the initial local excitation does not spread significantly.

Strictly speaking, while disordered 1D systems are always localized, there are two mechanisms contributing to localization. One of them is the "strong localization", i.e., the convergence, term by term, of a perturbation theory for the local Green's function. The other is the "weak localization", originated in the interferences between long perturbation pathways. This last one was an idea conceptually difficult to grasp, both theoretically and numerically, until the appearance of the scaling theory 
Papers in Physics, vol. 7, ART. 070012 (2015) / P. R. Zangara et al.

of conductance by the "gang of four" [48]. While weak localization is particularly relevant in $1 \mathrm{D}$ and 2D systems, when these have a finite size the dynamics remains diffusive and thus closely assimilable to an ergodic one. In our problem, as soon as $\Delta \gtrsim 0$, the many-body interaction increases the effective dimensionality of the available Hilbert space, and thus it competes with the Anderson localization. Regardless of the precise behavior near $\Delta=0$, such an interplay between $\Delta$ and $W$ is the responsible for the onset of a localization transition at some $W_{c}(\Delta)>0$, much as in a high dimensional lattice.

The ergodic-localized MBL transition can be observed when increasing $W$ for a fixed $\Delta>0$. In particular, we notice that localization by disorder is weakened when $1.0 \mathrm{~J} \lesssim \Delta \lesssim 2.0 \mathrm{~J}$, since the ergodic region seems to unfold for larger $W$. Again, since we consider a finite system, our observable describes a smooth crossover from the ergodic to a localized phase, where the excitation does not diffuse considerably. In fact, this corresponds to the actual MBL phase transition [27-31], which is genuinely sharp in the thermodynamic limit.

According to Eq. (7), $T \lesssim T_{H} \propto N$, and thus increasing $N$ in our simulations (e.g., 10, 12 and 14) enables an integration over a larger time $T$. In fact, when $\Delta \sim 1.0 J$, we verified that both sides of the MBL transition $\bar{M}_{1,1}$ behave as expected from physical grounds. Indeed, in the ergodic phase it has the asymptotic behavior $\bar{M}_{1,1} \sim 1 / N$, while in the localized phase of strong $W$ it saturates at $\bar{M}_{1,1} \sim 1 / \lambda$, regardless of $N$. The compatibility with a finite size scaling analysis is confirmed by the fact that $\partial \bar{M}_{1,1} / \partial W$ increases with $N$. However, our accessible range for $N$ is not complete enough to provide for a scaling of $\bar{M}_{1,1}(T)$ that could yield precise critical values for the MBL transition.

In analogy to the case of the Mott transition, a dimensional argument can be performed to estimate the critical line $W_{c}(\Delta)$. Even though there is no actual phase transition in the $1 \mathrm{D}$ non-interacting case $\Delta=0$, as the interactions appear we expect them to break down the 1D constrains. Thus, we consider as a singular point the high dimensional estimate that occurs when the disorder strength is comparable to the bandwidth [49]

$$
\left.W_{c}(\Delta)\right|_{\Delta=0}=(e / 2) B .
$$

The particular disorder strength in Eq. (12) is indicated in Fig. 1 as an open circle, since it does not correspond to an actual critical point of the $1 \mathrm{D}$ problem. Again, adding interactions would introduce an energy uncertainty that widens the band accordingly. In this case, the uncertainty $\delta E$ is associated to the lifetime introduced by the Ising interactions. The corresponding FGR evaluation for such a time-scale is explicitly performed in Ref. [50] and it yields:

$$
\frac{1}{\tau}=2 \frac{2 \pi}{\hbar} \Delta^{2} \frac{4}{3 \pi^{2} J} .
$$

As above, the extra 2 factor stands for the contributions of two semi-infinite linear chains. The factor $4 /\left(3 \pi^{2} J\right)$ stands for the corresponding LDoS evaluated at $\varepsilon=0$. Then,

$$
\delta E=\frac{\hbar}{2 \tau}=\frac{8}{3 \pi} \frac{\Delta^{2}}{J} .
$$

This uncertainty adds to the bandwidth and hence it leads to the dimensional estimation of the critical line of the MBL transition,

$$
\begin{aligned}
W_{c}(\Delta) & \sim \frac{e}{2}(B+2 \delta E) \\
& \sim \frac{2.71}{2}\left(2 J+\frac{16}{3 \pi} \frac{\Delta^{2}}{J}\right),
\end{aligned}
$$

which is plotted in Fig. 1 as a dashed line starting in the open circle.

\section{Conclusion}

We simulated the dynamics of a local Loschmidt echo in a spin system in the presence of interactions and disorder, for a wide regime of these competing magnitudes. The computation yields a phase diagram that evidences the parametric region where ergodicity manifests. Non-ergodic behaviors were classified and discussed in terms of glassy dynamics, standard Anderson Localization and the ManyBody Localization. Based on the evaluation of energy uncertainties introduced by weak interactions and weak disorder, we estimated the critical lines that separate these phases. The agreement between the estimated critical lines and the LE diagram is considerably good.

In spite of the fact that the local nature of the LE observable constitutes a limitation to perform 
PAPers in Physics, vol. 7, ART. 070012 (2015) / P. R. Zangara et al.

a reliable finite size scaling procedure, our strategy seems promising to analyze different underlying topologies and different ways of breaking down integrability. Last, but not least, in state-of-theart NMR [51,52], the high temperature correlation functions, like the LE, are privileged witnesses for the onset of phase transitions [53] that could hint the appearance of Many-Body Localization $[45,51,54]$.

Acknowledgements - PRZ and HMP wish to dedicate this paper to the memory of their coauthor Patricia Rebeca Levstein who did not live to see the final version of this paper. We are grateful to A. Iucci, A. D. Dente and C. Bederián for their cooperation at various stages of this work. This work benefited from fruitful discussions with T. Giamarchi and comments by F. Pastawski. We acknowledge support from CONICET, ANPCyT, SeCyT-UNC and MinCyT-Cor. The calculations were done on Graphical Processing Units under an NVIDIA Professor Partnership Program led by O. Reula.

[1] J L Lebowitz, Boltzmann's entropy and time's arrow, Phys. Today 46, 32 (1993).

[2] J L Lebowitz, Statistical mechanics: A selective review of two central issues, Rev. Mod. Phys. Supplement 71, 346 (1999).

[3] E Fermi, J Pasta, S Ulam, Studies of nonlinear problems, LASL Report LA1940 5, 977 (1955).

[4] E Fermi, Collected Pppers: United States 1939-1954, Vol. 2, University of Chicago Press (1965).

[5] G P Berman, F M Izrailev, The Fermi-PastaUlam problem: Fifty years of progress, Chaos 15, 015104 (2005).

[6] B V Chirikov, Resonance processes in magnetic traps, J. Nucl. Energy C 1, 253 (1960).

[7] F M Izrailev, B V Chirikov, Statistical properties of a nonlinear string, Sov. Phys. Dokl. 11, 30 (1966).
[8] G M Zaslavsky, Chaotic dynamics and the origin of statistical laws, Phys. Today 52, 39 (1999).

[9] S Goldstein, J L Lebowitz, R Tumulka, $\mathrm{N}$ Zanghì, Long-time behavior of macroscopic quantum systems. Commentary accompanying the English translation of John von Neumann's 1929 article on the quantum ergodic theorem, Eur. Phys. J. H 35, 173 (2010).

[10] J von Neumann, Proof of the ergodic theorem and the H-theorem in quantum mechanics. Translation of: Beweis des ergodensatzes und des H-theorems in der neuen mechanik, Eur. Phys. J. H 35, 201 (2010).

[11] R A Jalabert, H M Pastawski, Environmentindependent decoherence rate in classically chaotic systems, Phys. Rev. Lett. 86, 2490 (2001).

[12] P R Levstein, G Usaj, H M Pastawski, Attenuation of polarization echoes in nuclear magnetic resonance: A study of the emergence of dynamical irreversibility in many-body quantum systems, J. Chem. Phys. 108, 2718 (1998).

[13] A Goussev, R A Jalabert, H M Pastawski, D Wisniacki, Loschmidt echo, Scholarpedia $\mathbf{7}$, 11687 (2012).

[14] P R Zangara, A D Dente, P R Levstein, H M Pastawski, Loschmidt echo as a robust decoherence quantifier for many-body systems, Phys. Rev. A 86012322 (2012).

[15] Ph Jacquod, C Petitjean, Decoherence, entanglement and irreversibility in quantum dynamical systems with few degrees of freedom, Adv. in Phys. 58, 67 (2009).

[16] H M Pastawski, P R Levstein, G Usaj, J Raya, $\mathrm{J}$ Hirschinger, A nuclear magnetic resonance answer to the Boltzmann-Loschmidt controversy? Physica A 283, 166 (2000).

[17] G Usaj, H M Pastawski, P R Levstein, Gaussian to exponential crossover in the attenuation of polarization echoes in NMR, Mol. Phys. 95, 1229 (1998).

[18] T Kinoshita, T Wenger, D S Weiss, A quantum Newton's cradle, Nature 440, 900 (2006). 
Papers in Physics, vol. 7, ART. 070012 (2015) / P. R. Zangara et al.

[19] S Trotzky, Y-A Chen, A Flesch, I P McCulloch, U Schollwöck, J Eisert, I Bloch, Probing the relaxation towards equilibrium in an isolated strongly correlated one-dimensional Bose gas, Nat. Phys. 8, 325 (2012).

[20] A Polkovnikov, K Sengupta, A Silva, M Vengalattore, Colloquium: Nonequilibrium $d y$ namics of closed interacting quantum systems. Rev. Mod. Phys. 83, 863 (2011).

[21] D M Basko, I L Aleiner, B L Altshuler, Metal insulator transition in a weakly interacting many-electron system with localized singleparticle states, Ann. Phys. New York 321, 1126 (2006).

[22] I L Aleiner, B L Altshuler, G V Shlyapnikov, A finite-temperature phase transition for disordered weakly interacting bosons in one dimension, Nat. Phys. 6, 900 (2010).

[23] N F Mott, Metal-insulator transition, Rev. Mod. Phys. 40, 677 (1968).

[24] P W Anderson, Local moments and localized states, Rev. Mod. Phys. 50, 191 (1978).

[25] S Popescu, A J Short, A Winter,Entanglement and the foundations of statistical mechanics. Nat. Phys. 2, 754 (2006).

[26] M Rigol, V Dunjko, M Olshanii, Thermalization and its mechanism for generic isolated quantum systems, Nature 452, 854 (2008).

[27] V Oganesyan, D A Huse, Localization of interacting fermions at high temperature, Phys. Rev. B 75, 155111 (2007).

[28] M Žnidarič, T Prosen, P Prelovšek, Many-body localization in the Heisenberg $X X Z$ magnet in a random field, Phys. Rev. B 77, 064426 (2008).

[29] A Pal, D A Huse, Many-body localization phase transition, Phys. Rev. B 82, 174411 (2010).

[30] J H Bardarson, F Pollmann, J E Moore, Unbounded growth of entanglement in models of many-body localization, Phys. Rev. Lett. 109, 017202 (2012).
[31] A De Luca, A Scardicchio, Ergodicity breaking in a model showing many-body localization, Europhys. Lett. 101, 37003 (2013).

[32] D Pekker, G Refael, E Altman, E Demler, V Oganesyan, The Hilbert-glass transition: new universality of temperature-tuned many-body dynamical quantum criticality, Phys. Rev. X 4, 011052 (2014).

[33] T Giamarchi, H J Schulz, Anderson localization and interactions in one-dimensional metals, Phys. Rev. B 37, 325 (1988).

[34] C A Doty, D S Fisher, Effects of quenched disorder on spin-1/2 quantum XXZ chains, Phys. Rev. B 45, 2167 (1992).

[35] J Kimball, Comments on the interplay between Anderson localisation and electron-electron interactions, J. Phys. C Solid State 14, L1061 (1981).

[36] H M Pastawski, G Usaj, P R Levstein, Quantum interference phenomena in the local polarization dynamics of mesoscopic systems: an NMR observation, Chem. Phys. Lett. 261, 329 (1996).

[37] Z L Mádi, B Brutscher, T Schulte-Herbrüggen, R Brüschweiler, R R Ernst, Time-resolved observation of spin waves in a linear chain of nuclear spins, Chem. Phys. Lett. 268, 300 (1997).

[38] E P Danieli, H M Pastawski, P R Levstein, Spin projection chromatography, Chem. Phys. Lett. 384, 306 (2004).

[39] B Kramer, A MacKinnon, Localization: Theory and experiment, Rep. Prog. Phys. 56, 1469 (1993).

[40] G A Álvarez, E P Danieli, P R Levstein, $\mathrm{H}$ M Pastawski, Quantum parallelism as a tool for ensemble spin dynamics calculations, Phys. Rev. Lett. 101, 120503 (2008).

[41] S Zhang, B H Meier, R R Ernst, Polarization echoes in NMR, Phys. Rev. Lett. 69, 2149 (1992). 
Papers in Physics, vol. 7, ART. 070012 (2015) / P. R. Zangara et al.

[42] P Cappellaro, Implementation of state transfer Hamiltonians in spin chains with magnetic resonance techniques, In: Quantum State Transfer and Network Engineering, Eds. G M Nikolopoulos, I Jex, Pag. 183-222, Springer Berlin Heidelberg (2014).

[43] W-K Rhim, A Pines, J S Waugh, Timereversal experiments in dipolar-coupled spin systems, Phys. Rev. B 3, 684 (1971).

[44] E Rufeil-Fiori, C M Sánchez, F Y Oliva, H M Pastawski, P R Levstein, Effective one-body dynamics in multiple-quantum NMR experiments, Phys. Rev. A 79, 032324 (2009).

[45] G A Álvarez, D Suter, R Kaiser, Experimental observation of a phase transition in the evolution of many-body systems with dipolar interactions, arXiv:1409.4562 (2014).

[46] H M Pastawski, P R Levstein, G Usaj, Quantum dynamical echoes in the spin diffusion in mesoscopic systems, Phys. Rev. Lett. 75, 4310 (1995).

[47] L J Fernández-Alcázar, H M Pastawski, Decoherent time-dependent transport beyond the Landauer-Büttiker formulation: A quantumdrift alternative to quantum jumps, Phys. Rev. A 91, 022117 (2015).

[48] E Abrahams, P W Anderson, D C Licciardello, T V Ramakrishnan, Scaling theory of localiza- tion: Absence of quantum diffusion in two dimensions, Phys. Rev. Lett. 42, 673 (1979).

[49] J M Ziman, Localization of electrons in ordered and disordered systems ii. Bound bands, J. Phys. C Solid State 2, 1230 (1969).

[50] E P Danieli, G A Álvarez, P R Levstein, H M Pastawski, Quantum dynamical phase transition in a system with many-body interactions, Solid State Commun. 141, 422 (2007).

[51] M B Franzoni, P R Levstein, Manifestations of the absence of spin diffusion in multipulse NMR experiments on diluted dipolar solids, Phys. Rev. B 72, 235410 (2005).

[52] S W Morgan, V Oganesyan, G S Boutis, Multispin correlations and pseudothermalization of the transient density matrix in solidstate NMR: Free induction decay and magic echo,Phys. Rev. B 86, 214410 (2012).

[53] J Zhang, F M Cucchietti, C M Chandrashekar, M Laforest, C A Ryan, M Ditty, A Hubbard, J K Gamble, R Laflamme, Direct observation of quantum criticality in Ising spin chains, Phys. Rev. A 79, 012305 (2009).

[54] G A Álvarez, D Suter, NMR quantum simulation of localization effects induced by decoherence, Phys. Rev. Lett. 104, 230403 (2010). 